\title{
The MRTF-A/B function as oncogenes in pancreatic cancer
}

\author{
ZHAO SONG ${ }^{1}$, ZHAO LIU $^{1}$, JING SUN ${ }^{2}$, FENG-LEI SUN ${ }^{1}$, CHUAN-ZHI LI ${ }^{1}$, \\ JIU-ZHENG SUN ${ }^{1}$ and LI-YOU XU ${ }^{1}$ \\ Departments of ${ }^{1}$ Hepatobiliary and Pancreatic Surgery and ${ }^{2}$ Radiology, \\ Jinan Central Hospital Affiliated to Shandong University, Jinan, Shandong 250013, P.R. China
}

Received June 29, 2015; Accepted August 6, 2015

DOI: 10.3892/or.2015.4329

\begin{abstract}
Despite evidence that MRTF-A/B, co-activators of serum response factor (SRF), promotes tumor cell invasion and metastasis in cancer, there are no studies describing MRTF-A/B in pancreatic cancer. To clarify involvement of MRTF-A/B expression in pancreatic cancer, we used quantitative reverse transcription-polymerase chain reaction and western blot analysis to detect MRTF-A/B in pancreatic cancer, intraductal papillary mucinous neoplasm (IPMN) and non-neoplastic pancreata. MRTF-A/B expression differs significantly between cancer and non-neoplastic tissues as well as between non-neoplastic tissues and IPMN bulk tissues. Next, we studied the roles of MRTF-A/B in vitro. Overexpression of MRTF-A/B promoted epithelial-mesenchymal transition (EMT) and generated stem cell-like cells in normal pancreatic cells. We performed quantitative reverse transcription-polymerase chain reaction to detect the level of MRTF-A/B in 19 pancreatic cancer cell lines. We found that their expression was associated with gemcitabine resistance. Like in normal pancreatic cells, MRTF-A/B also promoted EMT and promoted formation of stem cell-like cells in pancreatic cancer and they could regulate microRNA expression associated with EMT and CICs. Finally, to further demonstrate the roles of MRTF-A/B in vivo, we performed nude mouse model of s.c. xenograft and found that overexpression of MRTF-A and MRTF-B promoted pancreatic cancer growth. Elucidating the roles of MRTF-A/B will help us to further understand
\end{abstract}

Correspondence to: $\mathrm{Dr} \mathrm{Li}-\mathrm{You} \mathrm{Xu}$, Department of Hepatobiliary and Pancreatic Surgery, Jinan Central Hospital Affiliated to Shandong University, 105 Jiefang Road, Jinan, Shandong 250013, P.R. China

E-mail: xuliyou0521sd@sina.com

Abbreviations: CICs, cancer-initiating cells; CSCs, cancer stem cells; EMT, epithelial-mesenchymal transition; IDC, invasive ductal carcinoma; IPMN, intraductal papillary mucinous neoplasm; MET, mesenchymal-epithelial transition; MRTFs, myocardin-related transcription factors

Key words: pancreatic cancer, myocardin-related transcription factors-A/B, cancer-initiating cells, epithelial-mesenchymal transition molecular basis of the disease and offer new gene targets for effective therapies.

\section{Introduction}

Pancreatic adenocarcinoma is a highly lethal disease and it is currently the fourth leading cause for cancer-related mortality $(1,2)$. The overall 5 -year survival rate among patients with pancreatic cancer is $<5 \%(2,3)$. The mortality rate approaches $100 \%$ due to the propensity for early metastatic spread and because the disease is highly resistant to radiation and chemotherapy (2). Increasing evidence indicates that human pancreatic cancer is driven by 'cancer-initiating cells (CICs)' popularly also known as 'cancer stem cells' (CSCs) that may contribute to tumor metastasis and therapeutic resistance (4-7). The molecular basis for these characteristics of CICs or CSCs is incompletely understood.

The transcription factor, serum response factor (SRF) is an important regulator of cytoskeletal and muscle-specific gene expression $(8,9)$. The myocardin family members MRTF-A/B (also known as megakaryocytic acute leukemia and megakaryoblastic leukemia-1/2) are well-established co-activators of SRF $(10,11)$. Although myocardin expression is restricted to smooth and cardiac muscle lineages, MRTF-A/B are expressed ubiquitously. The activity of MRTF-A/B is regulated by their translocation from the cytosol to the nucleus, which is triggered by the activation of Rho family proteins (11). Similar to myocardin, MRTF-A/B are reported to contribute to skeletal, cardiac and smooth muscle differentiation (12). For example, Wnt2 signaling activates the airway smooth muscle program in the lung by regulating myocardin/MRTF-B expression (13). Although their importance in mammary myoepithelial differentiation $(14,15)$ and the epithelial-mesenchymal transition (EMT) (16) has been established, their functions in cancer are completely different between myocardin and MRTF-A/B. Myocardin as an effective inducer of growth arrest and differentiation of certain tumors and is frequently repressed during human malignant transformation $(12,17)$. Yet, MRTF-A/B promotes tumor cell invasion and metastasis (18). Up to now, the roles of MRTF-A and MRTF-B have not been reported in pancreatic cancer.

Herein, to clarify involvement of MRTF-A/B expression in pancreatic cancer, we used quantitative reverse transcription-polymerase chain reaction and western blot analysis to 
detect MRTF-A/B in pancreatic cancer, intraductal papillary mucinous neoplasm (IPMN) and non-neoplastic pancreata. MRTF-A/B expression differed significantly between cancer and non-neoplastic tissues as well as also differed between non-neoplastic tissues and IPMN bulk tissues. Next, we studied the roles of MRTF-A/B in vitro. Overexpression of MRTF-A/B promoted EMT and generated stem cell-like cells in normal pancreatic cells. We performed quantitative reverse transcription-polymerase chain reaction to detect the level of MRTF-A/B in 19 pancreatic cancer cell lines. We found that their expression was associated with gemcitabine resistance. Like in normal pancreatic cells, MRTF-A/B also promoted EMT and generated stem cell-like cells in pancreatic cancer cells and they could regulate microRNA expression associated with EMT and CICs. Finally, to further demonstrate the roles of MRTF-A/B in vivo, we performed a nude mouse model of s.c. xenografts and found that overexpression of MRTF-A and MRTF-B promoted pancreatic cancer growth. Elucidating the roles of MRTF-A/B will help us to further understand molecular basis of the disease and offer new gene targets for effective therapies.

\section{Materials and methods}

Pancreatic tissues. Tissues were obtained from the primary tumor of each resected pancreas for a total of 37 IDC and 48 IPMN samples as well as 32 non-neoplastic pancreata at the time of surgery at the Department of Hepatobiliary and Pancreatic Surgery, Jinan Central Hospital and Hubei Cancer Hospital. All tissues were examined histologically, and pathologists confirmed the diagnosis. The University Medical Ethics Committee approved the experiments undertaken. The use of human tissue samples follows internationally recognised guidelines as well as local and national regulations. Informed consent was obtained from each individual.

Cell culture. HPDE6c7 normal pancreatic cells and pancreatic cancer cell lines KP-2, CFPAC-1, KP-3, H48N, Suit-2, Capan-1, SW1990, Kp-1N, L3.6pl, Colo357, BxPC-3, HPAC, CFPAC, Capan-2, MiaPaCa-2, NOR-P1, ASPC-1, PANC-1 and HS766T were obtained from the American Type Culture Collection (ATCC). HPDE6c7 normal pancreas cells as well as pancreatic cancer cell lines BxPC-3 and Colo357 were chosen to establish stable cell lines overexpressing MRTF-A and MRTF-B for this study. HPDE6c7, BxPC-3 and Colo357 cells were transfected with the corresponding empty vector pcDNA3 Neo or pcDNA3-MRTF-A or pcDNA3-MRTF-B by using ExGen-500 transfection reagent (Fermentas, Germany) following the manufacturer's instructions, as previously described $(1,19,20)$ and referred to as HPDE6c7-control or HPDE6c7-MRTF-A or HPDE6c7-MRTF-B, BxPC-3control or BxPC-3-MRTF-A or BxPC-3-MRTF-B cells, Colo357-control or Colo357-MRTF-A or Colo357-MRTF-B, respectively. Stable cell lines overexpressing MRTF-A or MRTF-B were selected by antibiotics, gentamycin, and the level of expression was confirmed by western blot analysis. The stably transfected cell lines were cultured in Dulbecco's modified Eagle's medium (DMEM; Invitrogen, Carlsbad, CA, USA), supplemented with $10 \%$ fetal bovine serum (FBS), $2 \mathrm{mmol} / \mathrm{l}$ glutamine, $50 \mathrm{U} / \mathrm{ml}$ penicillin, and $50 \mu \mathrm{g} / \mathrm{ml} \mathrm{strep-}$ tomycin. All cells were maintained in a $5 \% \mathrm{CO}_{2}$-humidified atmosphere at $37^{\circ} \mathrm{C}$.

Quantitative real-time RT-PCR ( $R R T-P C R)$. Quantitative real-time RT-PCR was described before (21). The specific primer sets for PCR were as follows: GAPDH forward, 5'-GAAGGTGAAGGTCGGAGTCA-3' and reverse, 5'-GAAG ATGGTGATGGGATTTC-3'; MRTF-A forward, 5'-ATGGA GCTGGTGGAGAAGAATATC-3' and reverse, 5'-GAAGGA GGAACTGTCTGCTACC-3'; MRTF-B, forward, 5'-CCAGA CCGCTCTGAGCTTG-3' and reverse, 5'-TCCTTGACACTC GAATCCAC3'; E-cadherin forward, 5'-TCAACGATCCTGA CCAGCAGTTCG-3' and reverse, 5'-GGTGAACCATCATC TGTGGCGATG-3'; N-cadherin forward, 5'-CATCCCTCCA ATCAACTTGC-3' and reverse, 5'-ATGTGCCCTCAAATG AAACC-3'; vimentin forward, 5'-GACAATGCGTCTCTGG CACGTCTT-3' and reverse, 5'-TCCTCCGCCTCCTGCAGG TTCTT-3'; ZEB1 forward, 5'-TTAGTTGCTCCCTGTGCA GTT-3' and reverse, 5'-TAGGAGCCAGAATGGGAAAAG-3'; FOXC2 forward, 5'-TCTCTCGCGCTCTCTCGCTC-3' and reverse, 5'-TGCAGCCCCTTAATTGTCTGG-3'; Slug, forward, 5'-TGATGAAGAGGAAAGACTACAG-3' and reverse, 5'-GCTCACATATTCCTTGTCACAG-3'; SIP1 forward, 5'-GTCCATGCGAACTGCCATCTGATCCGC TCT-3' and reverse, 5'-GGCTTGCAGAATCTCGCCAC-3'; TWIST, forward, 5'-GGAGTCCGCAGTCTTACGAG-3' and reverse, 5'-TCTGGAGGACCTGGTAGAGG-3'; and Snail forward, 5'-TCAGAATTCATGCCGCGCTCTTTCCTCGTC AGGAAGCC-3' and reverse, 5'-ACTGGATCCTCAGCGGG GACATCCTGAGCAGCCGGAC-3'.

Western blot analysis. Western blot analysis was performed as described before (22). Mainly, after incubation with primary antibody anti-MRTF-A (1:250); anti-MRTF-B (1:250); anti-CD44 (1:250); anti-Tspan8 (1:250); anti-c-Met (1:250); anti-CD151 (1:250); anti-ZEB1 (1:250); anti-Slug (1:250); anti-E-cad (1:250); anti-vimentin (1:250) and anti- $\beta$-actin (1:500) (all from Abcam, Cambridge, MA, USA) overnight at $4^{\circ} \mathrm{C}$. IRDye $\mathrm{T}^{\mathrm{TM}}-800$ conjugated anti-rabbit secondary antibodies (Li-COR Biosciences, Lincoln, NE, USA) were used for $30 \mathrm{~min}$ at room temperature. The specific proteins were visualized by Odyssey ${ }^{\mathrm{TM}}$ Infrared Imaging system (Gene Company, Lincoln, NE, USA).

Sphere growth. Cells $\left(10^{3} / \mathrm{ml}\right)$ in serum-free RPMI-1640/1 mM Na-pyruvate were seeded on $0.5 \%$ agar precoated 6 -well plates. After 1 week, half the medium was changed every third day. Single spheres were picked and counted.

Immunofluorescence analyses. For cell immunofluorescence analyses, cells were plated on glass coverslips in 6-well plates and transfected as indicated. At $48 \mathrm{~h}$ after transfection, coverslips were stained with the mentioned anti-fibronectin, anti-vimentin, anti-E-cadherin, and anti-EpCAM antibody. Alexa Fluor 488 goat anti-rabbit IgG antibody was used as secondary antibody (Invitrogen). Coverslips were counterstained with DAPI (Invitrogen-Molecular Probes, Eugene, Oregon, USA) for visualization of nuclei. Microscopic analysis was performed with a confocal laser-scanning microscope (Leica Microsystems, Bensheim, Germany). Fluorescence 
intensities were measured in a few viewing areas for 300 cells/coverslip and analyzed using ImageJ $1.37 \mathrm{v}$ software (http://rsb.info.nih.gov/ij/index.html).

Clonogenic assay. Cells were collected after trypsinization, and re-suspended in the complete medium. Single-cell suspensions were plated in regular $10 \mathrm{~cm}$ in diameter Petri dishes at the clonal density of 1,000 cells/dish. After 3 weeks of culture, colonies were fixed with $4 \%$ paraformaldehyde for $10 \mathrm{~min}$, stained with crystal violet for additional $15 \mathrm{~min}$, and washed with $1 \mathrm{X}$ PBS. The colonies were photographed. The colony numbers were counted using software image analysis program Scion Image downloaded from NIH website (http://www. scioncorp.com). Particle Analysis program was used for counting the colony numbers. Data are presented as relative colony number.

Soft agar assay. Cells were harvested and suspended in culture medium. To make the bottom layer, $1 \mathrm{ml}$ of $0.5 \%$ agarose (Invitrogen) was added to 6 -well plates, and allowed to gel at room temperature. To prepare the top layer $(0.25 \%$ agarose), $500 \mu \mathrm{l}$ of $0.5 \%$ agarose was mixed with $500 \mu \mathrm{l}$ cell suspension containing the 5,000 cells. This mixture was laid above the bottom layer and allowed to solidify at room temperature. An additional $2 \mathrm{ml}$ of culture medium was added after solidification to the top layer, and cells were incubated for 4 weeks at $37^{\circ} \mathrm{C}$. After three weeks of growth, the colonies were photographed $(\mathrm{x} 40)$. The colony numbers were counted under a phase contrast microscope (x40). Data are presented as colony numbers per field.

S.C. tumor models. BxPC-control and BxPC-MRTF-A and BxPC-MRTF-B cells were harvested by trypsinization, and resuspended in RPMI-1640. Only single-cell suspensions with $>95 \%$ viability were used. The cells $\left(5 \times 10^{6}\right)$ were inoculated into the right flank (s.c. tumor model) of 5 to 6 -week-old male nude mice (NCI/Charles River Breeding Labs, Wilmington, MA, USA). All mice were cared for in accordance with the Office for Protection from Research Risks and Animal Welfare Act guidelines under an animal protocol approved by the Jinan Central Hospital of Medicine Institutional Animal Care and Use Committee. For the s.c. tumor model, the tumor size was measured weekly using a digital caliper (VWR International, Leuven, Belgium), and the tumor volume was calculated using the following formula: tumor volume $\left(\mathrm{mm}^{3}\right)=0.52 \mathrm{x}$ [width $(\mathrm{mm})]^{2} \mathrm{x}$ [length $\left.(\mathrm{mm})\right](23)$. After week 5, all surviving mice were euthanized with an overdose of $\mathrm{CO}_{2}$ exposure and evaluated for the presence of tumors and the metastases in their abdominal cavity. For both s.c. experiments, the animals were euthanized when their tumor size reached $2 \mathrm{~cm}$ in diameter or the animals became moribund during the observation period, and the time of euthanization was recorded as the time of mortality.

miRNA microarray. Total RNA from cultured cells, with efficient recovery of small RNAs, was isolated using the mirVana miRNA Isolation kit (Ambion, Austin, TX, USA). cRNA for each sample was synthesized by using 3' IVT Express kit (Affymetrix, Santa Clara, CA, USA) according to the manufacturer's instructions. The purified cRNA was fragmented by incubation in fragmentation buffer (provided in the 3' IVT Express kit) at $95^{\circ} \mathrm{C}$ for $35 \mathrm{~min}$ and chilled on ice. The fragmented labeled cRNA was applied to MicroRNA 2.0 array and hybridized in Genechip hybridization oven 640 (both from Affymetrix) at $45^{\circ} \mathrm{C}$ for $18 \mathrm{~h}$. After washing and staining in Genechip fluidics station 450, the arrays were scanned by using Genechip scanner 3000 (both from Affymetrix). The gene expression levels of the samples were normalized and compared by using Partek GS 6.5 (Partek, Inc., St. Louis, MO, USA). Average-linkage hierarchical clustering of the data was applied by using the Cluster; http://rana.lbl.gov) and the results were displayed by using TreeView (both: Eisen et al, Stanford, Stanford University, CA, USA; http:// rana.lbl.gov).

\section{Results}

Analysis of MRTF-A and MRTF-B expression in pancreatic cancer, IPMN, and non-neoplastic bulk tissues. We measured the levels of MRTF-A and MRTF-B in bulk tissues from 37 pancreatic cancers (conventional IDC), 48 IPMNs and 32 non-neoplastic pancreata. To normalize MRTF-A and MRTF-B expression, we used U6 as a reference gene. Pancreatic cancer bulk tissues expressed higher levels of MRTF-A (Fig. 1A) and MRTF-B (Fig. 1B) than did IPMN bulk tissues (pancreatic cancer vs. IPMN, p<0.05), there was a significant difference between pancreatic cancer and nonneoplastic pancreata $(\mathrm{p}<0.01)$. We also observed that IPMN bulk tissues expressed higher levels of MRTF-A and MRTF-B than non-neoplastic pancreata (IPMN bulk tissues vs. nonneoplastic pancreata, $\mathrm{p}<0.05)$. Representative results of electrophoresis of RT-PCR products are shown in the bottom of Fig. 1A and B. Next, we performed western blot analysis to detect MRTF-A and MRTF-B level among non-neoplastic pancreas, IPMN and pancreatic cancer. Consistent with the results of RT-PCR, the results of western blot analysis showed that MRTF-A(Fig. 1C) and MRTF-B (Fig. 1D) were upregulated in pancreatic cancer, compared with IPMN and non-neoplastic pancreas. We also found that IPMN bulk tissues expressed higher levels of MRTF-A (Fig. 1C) and MRTF-B (Fig. 1D) than did non-neoplastic pancreas (IPMN bulk tissues vs. non-neoplastic pancreata, $\mathrm{p}<0.05)$. Representative results of western blot analysis are shown (Fig. 1C and D). The results implied that upregulation of MRTF-A and MRTF-B are associated with the development of pancreatic cancer and IPMN.

Analysis of MRTF-A and MRTF-B expression in a normal pancreas cell line and pancreatic cancer cell lines and their expression associated with gemcitabine resistance. We also performed real-time PCR to detect the expression of MRTF-A and MRTF-B in the HPDE6C7 normal pancreatic cell line and 19 pancreatic cancer cell lines. Except for KP2 cells, we detected MRTF-A and MRTF-B mRNA in 18 pancreatic cancer cell lines (Table I). It has been reported that L3.6pl, Colo357, BxPC-3, HPAC and CFPAC cells are gemcitabine-sensitive and MiaPaCa-2, ASPC-1, PANC-1 and HS766T cells are gemcitabine-resistant $(24,25)$. We found that expression of MRTF-A and MRTF-B was significantly increased in gemcitabine-resistant cells (Table I). To confirm that MRTF-A and MRTF-B were increased in gemcitabine-resistant cells, 
A

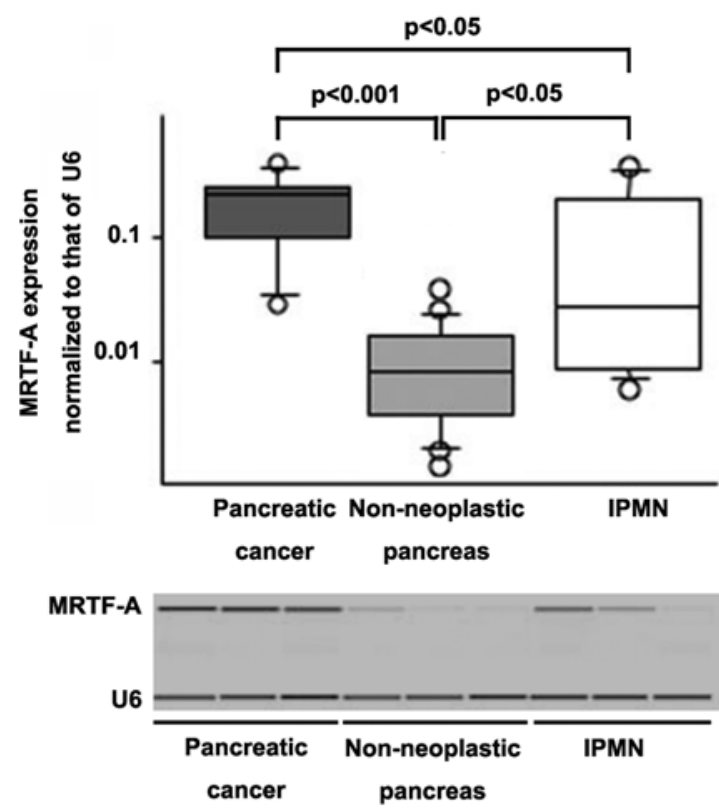

C

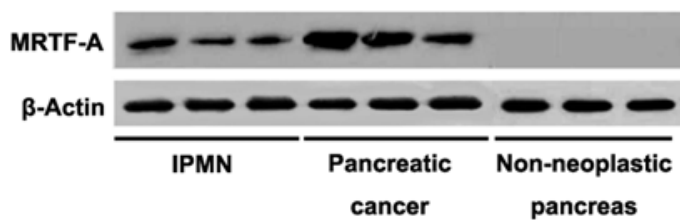

B

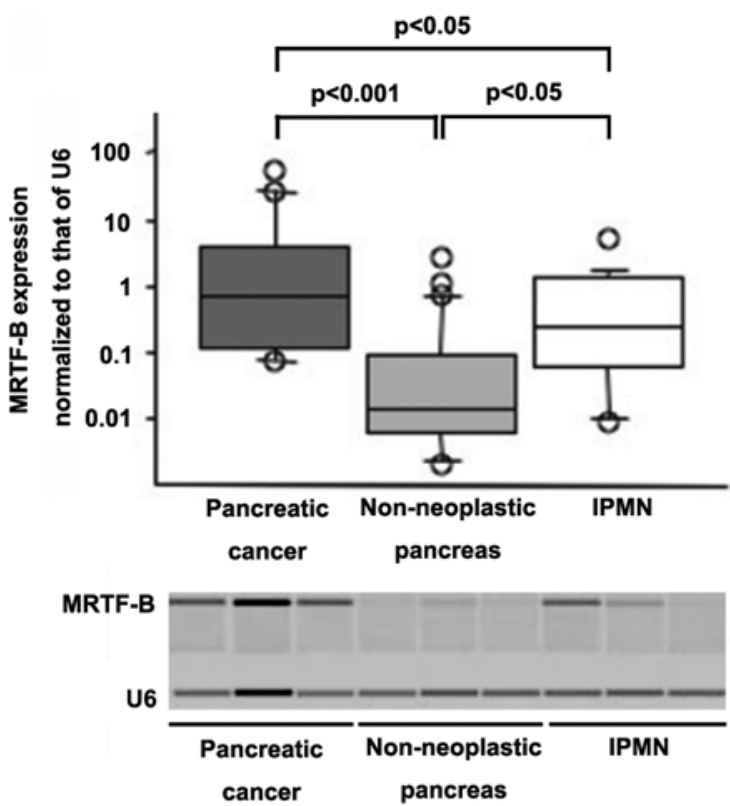

D

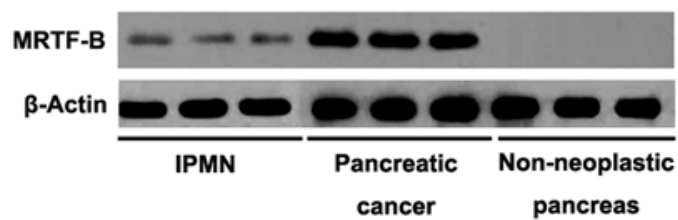

Figure 1. Expression of MRTF-A and MRTF-B in pancreatic cancer, IPMN, and non-neoplastic pancreatic bulk tissues. (A) Upper panel shows that there were significant differences in expression of MRTF-A between pancreatic cancer and IPMN $(p<0.05)$, between pancreatic cancer and non-neoplastic pancreas $(p<0.001)$ and between IPMN and non-neoplastic pancreas $(p<0.05)$ after Bonferroni's correction (upper panel). Lower panel shows a representative gel of the RT-PCR products. U6 was a loading control; $n=151$. (B) Upper panel shows that there were significant differences in expression of MRTF-B between pancreatic cancer and IPMN, between pancreatic cancer $(\mathrm{p}<0.05)$ and non-neoplastic pancreas $(\mathrm{p}<0.001)$ and between IPMN and non-neoplastic pancreas $(\mathrm{p}<0.05)$ after Bonferroni's correction (upper panel). Lower panel shows a representative gel of the RT-PCR products. U6 was a loading control; $\mathrm{n}=151$. (C) Western blot analysis for MRTF-A protein in pancreatic cancer, intraductal papillary mucinous neoplasm (IPMN), and non-neoplastic pancreatic bulk tissues. A representative gel of the western blot products. $\beta$-actin was a loading control; $n=143$. (D) Western blot analysis for MRTF-B protein in pancreatic cancer, IPMN, and non-neoplastic pancreatic bulk tissues. A representative gel of the western blot products. $\beta$-actin was a loading control; $n=143$. IPMN, intraductal papillary mucinous neoplasm; MRTF, myocardin-related transcription factor.

we performed western blot analysis to detect their protein. The results showed that MRTF-A and MRTF-B protein were evidently upregulated in gemcitabine-resistant cells (Fig. 2).

MRTF-A and MRTF-B promote EMT and generate stem cell-like cells in normal pancreatic cells. To determine whether MRTF-A and MRTF-B could promote EMT, HPDE6C7 cell line was chosen to establish a stable cell line overexpressing MRTF-A and MRTF-B for this study. HPDE6C7 cells were transfected with the corresponding empty vector pcDNA3 NEO or pcDNA3-MRTF-A or pcDNA3-MRTF-B. We performed real-time PCR and western blot analysis to detect MRTF-A in HPDE6C7MRTF-A cells. Compared with HPDE6C7-control cells, we found that MRTF-A mRNA (Fig. 3A) and its protein (Fig. 3B) were significantly upregulated in HPDE6C7-MRTF-A cells. Similar to HPDE6C7-MRTF-A cells, MRTF-B mRNA (Fig. 3C) and its protein (Fig. 3D) were significantly upregulated in HPDE6C7-MRTF-B cells.

We observed phase-contrast images of HPDE6C7 cells expressing the control vector, MRTF-A and MRTF-B, as well as HPDE6C 7 cells treated with TGF $\beta 1$ for 12 days, a standard treatment that induces EMT (26). The results showed that MRTF-A, MRTF-B and TGF $\beta 1$ induced EMT phenotype from a cobblestone-like to a spindle-like morphology (Fig. 3E), accompanied by increase of invasion and migration in the cells (data not shown). We also performed real-time PCR to detect epithelial and mesenchymal markers. As anticipated, HPDE6c7-MRTF-A and HPDE6c7-MRTF-B cells as well as HPDE6C7 cells treated with TGF $\beta 1$, epithelial markers (E-cadherin) was downregulated and mesenchymal markers (such as N-cadherin, vimentin, and Snail) were upregulated in HPDE6c7-MRTF-A and HPDE6c7-MRTF-B cells (Fig. 3F), compared with HPDE6c7-control cells. CD44, Tspan8, c-Met and CD151 are markers for CICs in pancreatic cancer $(5,27-30)$. We performed western blot analysis to detect the expression of CD44, Tspan8, c-Met and CD151 in MRTF-A and MRTF-B overexpressing pancreatic cancer cells. We found that the overexpression of MRTF-A and MRTF-B significantly upregulated CD44, Tspan8 and CD151 expression (Fig. 3G).

Following induction of EMT in HPDE6c7 cells by exposing them to TGFb1, the cells formed at least 40-fold more spheres than the untreated control cells (Fig. 3H). With these 
Table I. MRTF-A and MRTF-B expression in normal pancreas and pancreatic cancer cells.

\begin{tabular}{lcc}
\hline Pancreatic cancer cells & $\begin{array}{c}\text { MRTF-A } \\
\text { expression }\end{array}$ & $\begin{array}{c}\text { MRTF-B } \\
\text { expression }\end{array}$ \\
\hline KP-2 & ND $^{\mathrm{b}}$ & ND $^{\mathrm{b}}$ \\
HPDE6c7 & 0.001 & 0.001 \\
CFPAC-1 & 0.056 & 0.043 \\
KP-3 & 0.089 & 0.230 \\
H48N & 0.560 & 0.093 \\
Suit-2 & 0.091 & 0.045 \\
Capan-1 & 0.923 & 0.923 \\
SW1990 & 0.044 & 0.054 \\
Kp-1N & 0.027 & 0.076 \\
L3.6pl & 0.004 & 0.012 \\
Colo357 & 0.003 & 0.006 \\
BxPC-3 & 0.007 & 0.002 \\
HPAC & 0.010 & 0.009 \\
CFPAC & 0.008 & 0.125 \\
Capan-2 & 0.720 & 0.810 \\
MiaPaCa-2 & 0.810 & 0.576 \\
NOR-P1 & 0.110 & 0.231 \\
ASPC-1 & 0.990 & 0.990 \\
PANC-1 & 0.692 & 0.819 \\
HS766T & 0.786 & 0.996 \\
\hline MRTF-A & &
\end{tabular}

${ }^{a}$ MRTF-A and MRTF-B mRNA expression were normalized to that of $\mathrm{U} 6$; ${ }^{\mathrm{N} D}$, not detected; MRTF, myocardin-related transcription factor.

findings in mind, we examined the sphere forming ability of the HPDE6C7 cells forced to undergo EMT by ectopic expression of MRTF-A or MRTF-B. Significantly, we found that the HPDE6c7-MRTF-A and HPDE6c7-MRTF-B cells that underwent EMT formed $>60$-fold more spheres than the cells infected with the corresponding control vector (Fig. 3I). Based on this functional assay, we concluded that overexpression of MRTF-A and MRTF-B was able to promote EMT and generate stem cell-like cells in normal pancreatic cells.

MRTF-A and MRTF-B promote EMT in pancreatic cancer. Having demonstrated that MRTF-A and MRTF-B promoted EMT and generated stem cell-like cells in normal pancreatic cells and MRTF-A and MRTF-B could promote metastasis in tumor cell and xenografts (18), we reasoned that MRTF-A and MRTF-B also could promote EMT in pancreatic cancer cells.

Because expression of MRTF-A and MRTF-B was low in BxPC-3 and Colo357 cells, these cells were chosen to establish the stable cell lines overexpressing MRTF-A and MRTF-B. BxPC-3 and Colo357 cells were transfected with the corresponding empty vector pcDNA3 NEO or pcDNA3-MRTF-A or pcDNA3-MRTF-B. We performed real-time PCR and western blot analysis to detect MRTF-A in BxPC-3-MRTF-A cells. Compared with BxPC-3-control cells, we found that MRTF-A mRNA (Fig. 4A) and its protein (Fig. 4B) were significantly upregulated in BxPC-3-MRTF-A cells. Real-time
A

- Gemcitabine - resistant pancreatic cancer cell lines

- Gemcitabine-sensitive pancreatic cancer cell lines

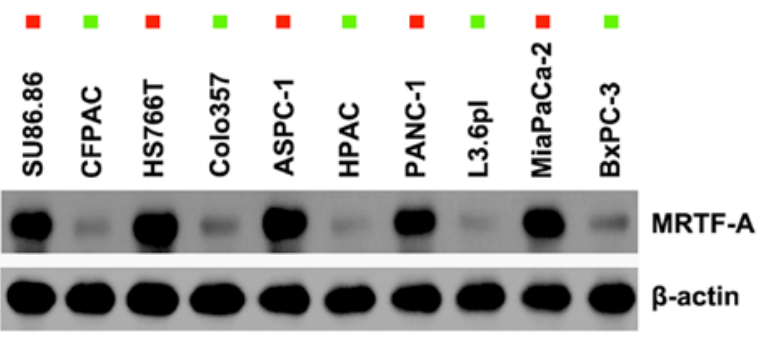

B

- Gemcitabine - resistant pancreatic cancer cell lines

- Gemcitabine-sensitive pancreatic cancer cell lines

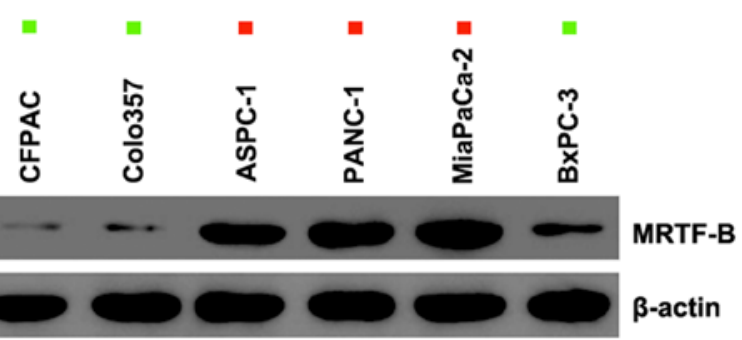

Figure 2. Expression of MRTF-A and MRTF-B in pancreatic cancer cells were associated with gemcitabine resistance. (A) Western blot analysis for MRTF-A protein in different pancreatic cancer cell lines. Representative gel of the western blot products was showed. $\beta$-actin was a loading control; $n=3$. (B) Western blot analysis for MRTF-B protein in different pancreatic cancer cell lines. Representative gel of the western blot products is shown. $\beta$-actin was a loading control; $n=3$. MRTF, myocardin-related transcription factor.

PCR and western blot analysis were also performed to detect MRTF-B mRNA and protein expression. The result showed that MRTF-B mRNA (Fig. 4C) and its protein (Fig. 4D) were significantly upregulated in BxPC-3-MRTF-B cells. Similar to BxPC-3 cells, MRTF-A (Fig. 4E and F) and MRTF-B (Fig. 4G and $\mathrm{H}$ ) were significantly increased in Colo357-MRTF-A and Colo357-MRTF-B cells, respectively.

In order to assess the role of MRTF-A and MRTF-B in pancreatic cancer, we observed phase-contrast images of pancreatic cancer BxPC-3 cells expressing the control vector, MRTF-A and MRTF-B. The results showed that MRTF-A and MRTF-B induced EMT phenotype from a cobblestone-like to a spindle-like morphology (Fig. 5A), accompanied by the decrease of E-cadherin expression and the increase of fibronectin and vimentin in the cells (Fig. 5B). We performed real-time PCR to detect epithelial marker (E-cadherin) and mesenchymal markers (N-cadherin, vimentin, ZEB1, FOXC2, Slug, SIP1, Twist and Snail). The results showed that epithelial marker (E-cadherin) was downregulated and all the mesenchymal markers were upregulated in MRTF-A or MRTF-B overexpressing BxPC-3 cells, compared with BxPC-3-control cells (Fig. 5C). Relative to the expression in BxPC-3-control cells, the level of the E-cadherin mRNA in BxPC-3-MRTF-A and BxPC-3-MRTF-B cells was strongly reduced (>100-fold), while expression of mRNAs encoding mesenchymal markers was markedly increased, specifically $\mathrm{N}$-cadherin (145- and 150-fold, respectively), vimentin (138- and 129-fold, respectively). There was also a considerable increase in the expression 
A

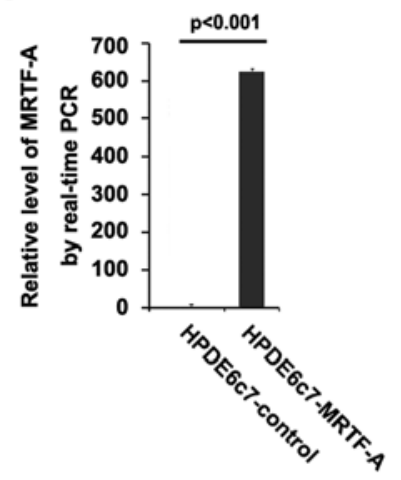

B

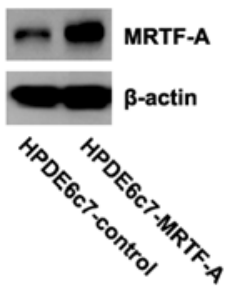

C

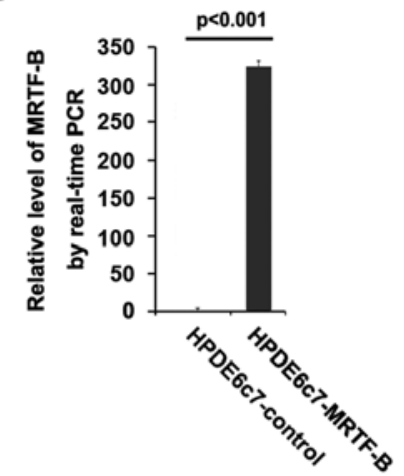

D

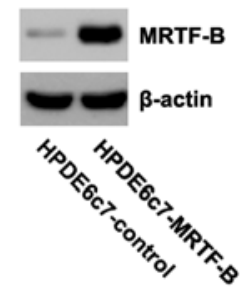

E

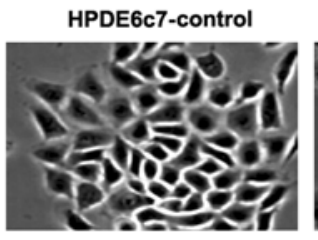

HPDE6c7-MRTF-B

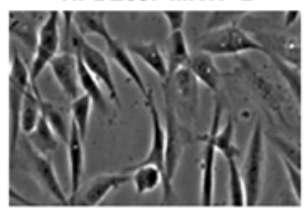

G

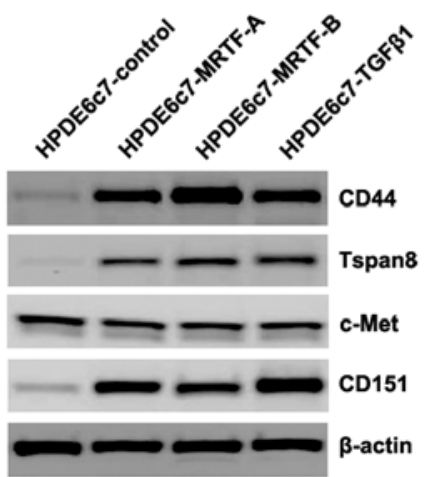

HPDE6c7-MRTF-A

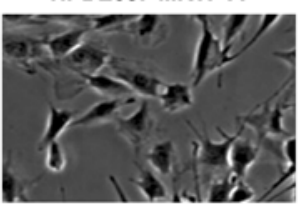

HPDE6c7-TGFß1

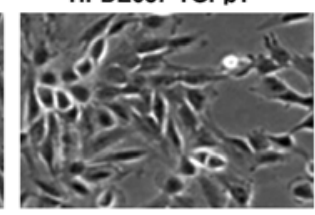

$\mathbf{F}$

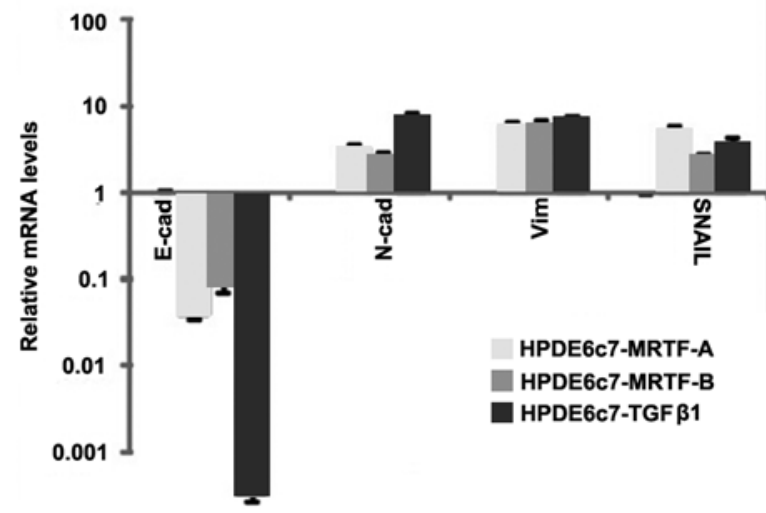

H

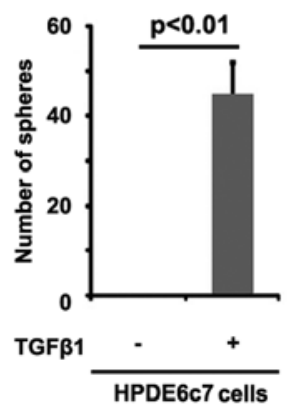

I

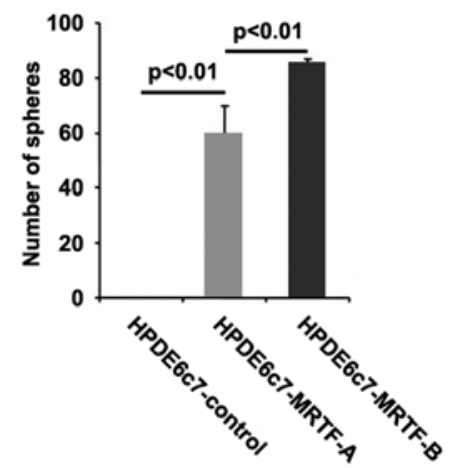

Figure 3. MRTF-A and MRTF-B generate EMT with properties of stem cells in normal pancreatic cells. (A) Real-time PCR for MRTF-A in HPDE6c7-control and HPDE6c7-MRTF-A cells. GAPDH was a loading control; $n=3$. (B) Western blot analysis for MRTF-A in HPDE6c7-control and HPDE6c7-MRTF-A cells. $\beta$-actin was a loading control; $n=3$. (C) Real-time PCR for MRTF-B in HPDE6c7-control and HPDE6c7-MRTF-B cells. GAPDH was a loading control; $n=3$. (D) Western blot analysis for MRTF-B in HPDE6c7-control and HPDE6c7-MRTF-B cells. $\beta$-actin was a loading control; n=3. (E) Phase-contrast images of HPDE6c7-control, HPDE6c7-MRTF-A and HPDE6c7-MRTF-B cells as well as HPDE6c7-control cells treated with recombinant TGF $\beta 1$ (2.5 ng/ml) for 12 days (bottom right); $n=3$. (F) Relative expression of the mRNAs encoding E-cadherin, N-cadherin, vimentin, and Snail in HPDE6c7-MRTF-A, HPDE6c7-MRTF-B, and HPDE6c7-control cells induced to undergo EMT, as determined by real-time RT-PCR. GAPDH mRNA was used to normalize the variability in template loading. The data are reported as mean \pm SEM; $n=3$. (G) Western blot analysis for CD44, Tspan8, c-Met and CD151 in BxPC-3 cells overexpressing pcDNA3 NEO, MRTF-A and MRTF-B as well as treated with TGF $\beta$. $\beta$-actin was a loading control; $\mathrm{n}=3$. (H) In vitro quantification of spheres formed by cells described in (E). The data are reported as the number of spheres formed/1,000 seeded cells \pm SEM; $n=3$. (I) In vitro quantification of spheres formed by cells described in (E). The data are reported as the number of spheres formed/1,000 seeded cells \pm SEM; $n=3$. EMT, epithelial-mesenchymal transition; MRTF, myocardin-related transcription factor.

of EMT-inducing transcription factors, specifically FOXC2 (62- and 129-fold), SIP1 (75- and 70-fold, respectively), Snail (49- and 74-fold, respectively), and Twist (140- and 220-fold); Slug expression was only marginally increased (5-fold) in MRTF-A overexpressing BxPC-3 cells, but it was significantly upregulated in BxPC-MRTF-B cells. To further confirm that the markers associated with EMT were affected by MRTF-A and MRTF-B, we performed western blot analysis to detect E-cadherin, ZEB1, vimentin and Slug protein. The result showed that MRTF-A and MRTF-B downregulated E-cadherin expression and upregulate ZEB1, vimentin and Slug expression in BxPC-3 cells (Fig. 5D). These measure- 
A

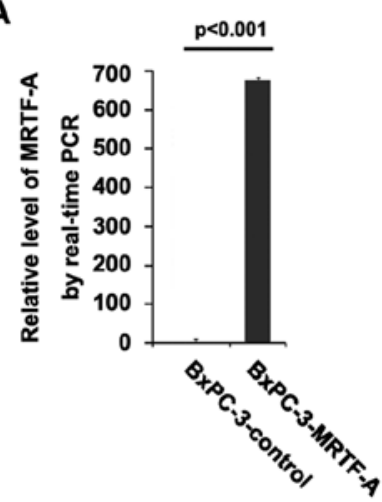

B

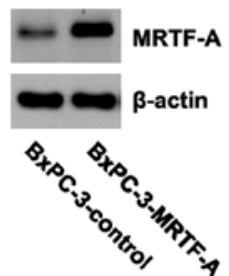

C

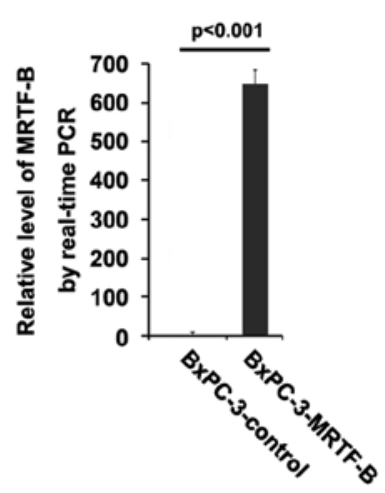

G

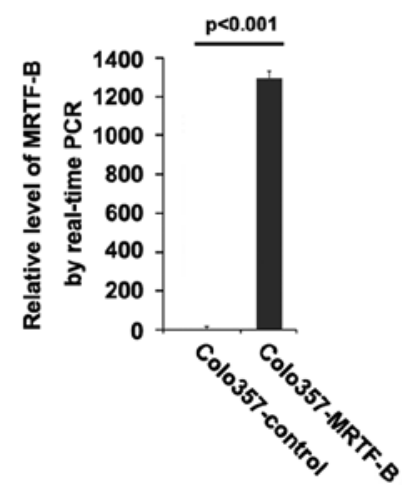

D
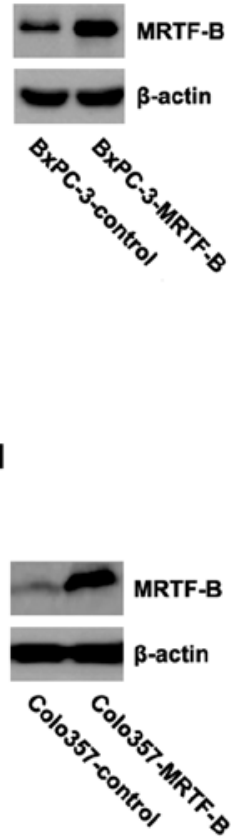
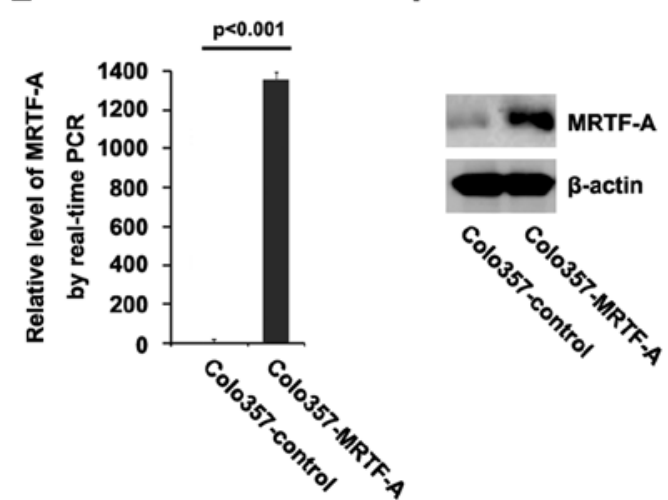

Figure 4. MRTF-A and MRTF-B expression are increased in BxPC-3-MRTF-A, BxPC-3-MRTF-B, Colo357-MRTF-A and Colo357-MRTF-B cells, respectively. (A) Real-time PCR for MRTF-A in BxPC-3-control and BxPC-3-MRTF-A cells. GAPDH was a loading control; $n=3$. (B) Western blot analysis for MRTF-A in BxPC-3-control and BxPC-3-MRTF-A cells. $\beta$-actin was a loading control; $n=3$. (C) Real-time PCR for MRTF-B in BxPC-3-control and BxPC-3-MRTF-B cells. GAPDH was a loading control; $n=3$. (D) Western blot analysis for MRTF-B in BxPC-3-control and BxPC-3-MRTF-B cells. $\beta$-actin was a loading control; $n=3$. (E) Real-time PCR for MRTF-A in Colo357-control and Colo357-MRTF-A cells. GAPDH was a loading control; $n=3$. ( $F$ ) Western blot analysis for MRTF-A in Colo357-control and Colo357-MRTF-A cells. $\beta$-actin was a loading control; $n=3$. (G) Real-time PCR for MRTF-B in Colo357control and Colo357-MRTF-B cells. GAPDH was a loading control; n=3. (H) Western blot analysis for MRTF-B in Colo357-control and Colo357-MRTF-B cells. $\beta$-actin was a loading control; $\mathrm{n}=3$. MRTF, myocardin-related transcription factor.

ments provided further indication that MRTF-A and MRTF-B promote EMT in pancreatic cancer.

In addition, real-time PCR was performed to detect whether MRTF-A and MRTF-B overexpression could affect E-cadherin, N-cadherin, vimentin, Slug and Twist expression in Colo357 cells. The results showed that MRTF-A and MRTF-B downregulated E-cadherin and upregulated $\mathrm{N}$-cadherin, vimentin, Slug and Twist expression in the cells (Fig. 5E and F).

MRTF-A and MRTF-B promotes CSC in pancreatic cancer cells. To determine whether cells with EMT phenotype could have stem-like cell characteristics. Sphere forming assay was conducted to assess the capacity of CSC or CSC-like cell self renewal in this study. We found that BxPC-MRTF-A and BxPC-MRTF-B cells showed increased formation of spheres (Fig. 6A) consistent with increased expression of CSC surface marker, EpCAM in spheres of BxPC-MRTF-A and BxPC-MRTF-B cells compared to BxPC-control cells (Fig. 6B), suggesting that overexpression of MRTF-A and MRTF-B could increase the formation of pancreatospheres associated with increased expression of EpCAM, which further suggest that MRTF-A and MRTF-B is involved in the regulation of the CSC phenotype.
Next, we performed clonogenic assays. We found that clonogenic ability was significantly increased in BxPC3-MRTF-A and BxPC-3-MRTF-B cells compared with BxPC-3-control cells (Fig. 6C, upper panel). Fig. 6C, bottom panel, further indicated that BxPC-3-MRTF-A and BxPC-3MRTF-B cells showed $\sim 10$-fold increase in colony numbers relative to BxPC-3-control cells.

Soft agar assay, also known as anchorage-independent growth assay, was also performed. BxPC-3-MRTF-A and BxPC-3-MRTF-B cells showed dramatic increase in clonogenic potential compared to BxPC-3-control cells (Fig. 6D, upper panel). Fig. 6D, bottom panel, clearly shows the colony numbers generated from BxPC-3-control cells, BxPC-3-MRTF-A and BxPC-3-MRTF-B cells per microscopic field. These results demonstrated an increase in clonogenic ability of EMT cells. Colo357-MRTF-A and Colo357-MRTF-B cells also displayed an increased clonogenic capacity, compared with Colo357control cells, respectively (data not shown).

The effect of MRTF-A and MRTF-B on miRNA expression was identified by miRNA profiling in BxPC-3 cells. We next examined the ability of MRTF-A and MRTF-B to regulate miRNA expression by microarray analysis of miRNAs expression profiles in BxPC-3 cells. RNAs isolated from BxPC-3-control, 
A

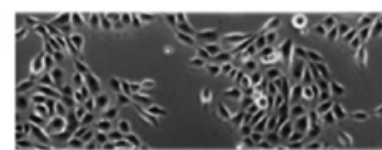

BxPC-3-control

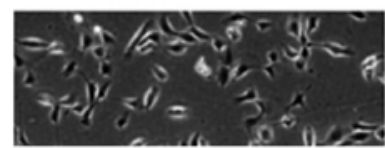

BXPC-3-MRTF-A

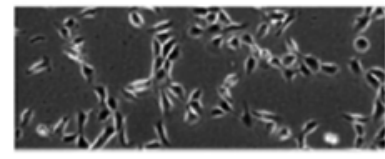

BXPC-3-MRTF-B
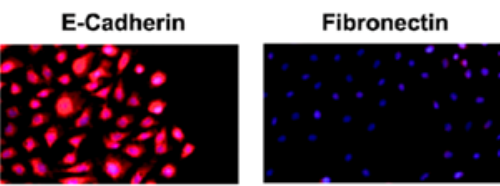

BxPC-3-control

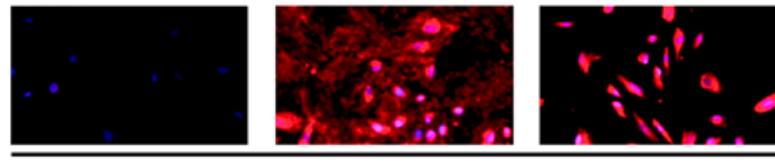

BXPC-3-MRTF-A

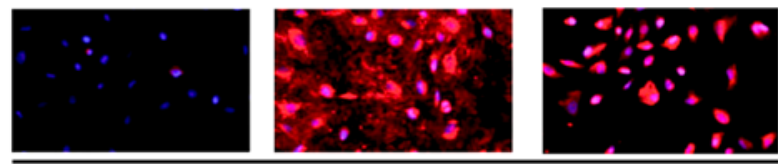

BXPC-3-MRTF-B

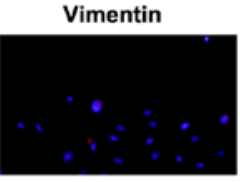

C

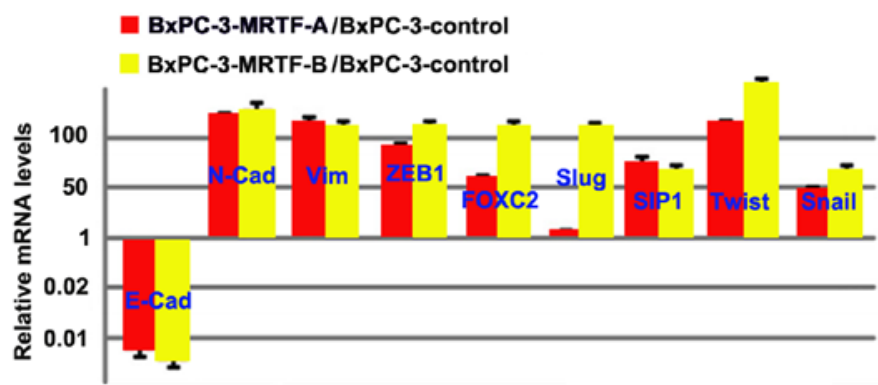

D

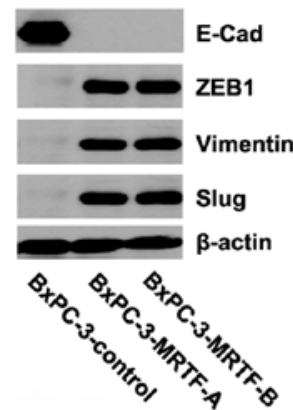

E

$\mathbf{F}$ 
A
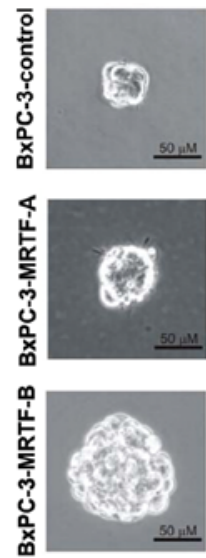

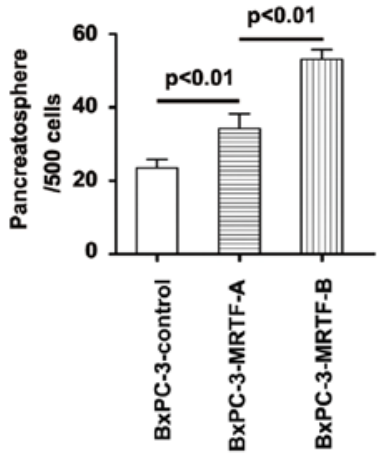

C

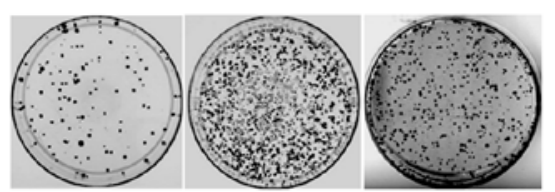

BxPC-3-control BxPC-3-MRTF-A BxPC-3-MRTF-B

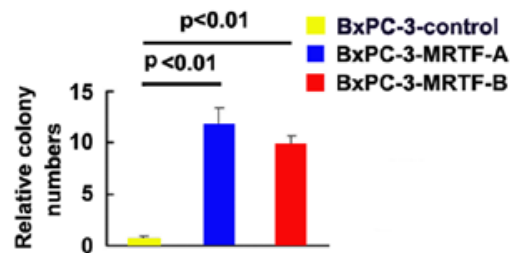

B

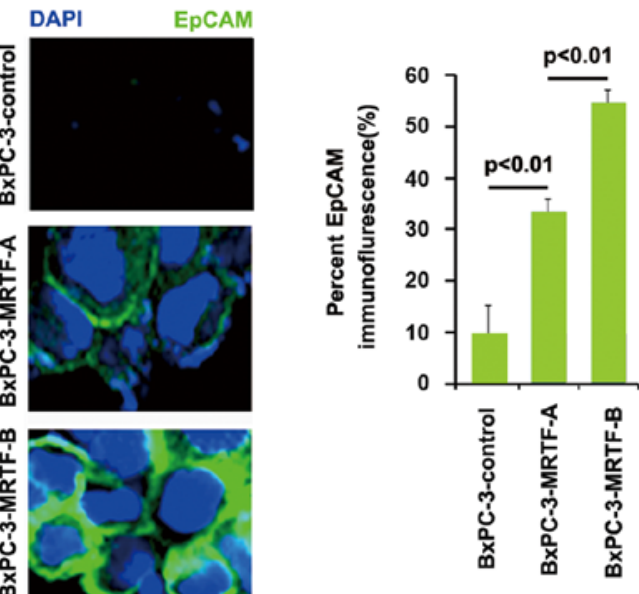

D
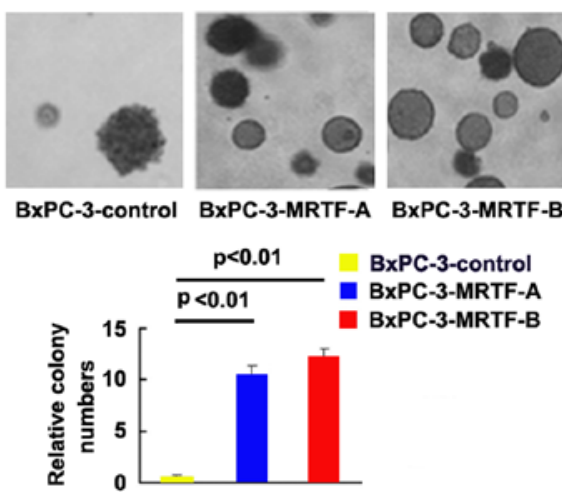

Figure 6. MRTF-A and MRTF-B promotes CSC in pancreatic cancer cells. (A) Sphere growth for BxPC-3-control, BxPC-3-MRTF-A and BxPC3 -MRTF-B cells. Left panel shows microscopic pictures of sphere growth. Right panel shows graphic presentation of mean no. of sphere growth; $\mathrm{n}=3$. (B) Immunofluorescence analyses for EpCAM in spheres of BxPC-3-control, BxPC-3-MRTF-A and BxPC-3-MRTF-B cells. Left panel shows microscopic pictures of immunofluorescence staining of one representative experiment (x100 magnifications). Right panel shows graphic presentation of mean fluorescence intensities; $n=3$. (C) Photographs of colonies from BxPC-3-control cells, BxPC-3-MRTF-A cells and BxPC-3-MRTF-B cells are shown (upper panel). The colony numbers were counted and the data are presented as relative colony numbers (bottom panel); $\mathrm{n}=3$. (D) The colonies of BxPC-3-control, BxPC-3MRTF-A and BxPC-3-MRTF-B cells grown on soft agar (upper panel). The colony numbers were counted under a phase contrast microscope. Data are presented as colony numbers per field (bottom panel); $n=3$. MRTF, myocardin-related transcription factor; CSC, cancer stem cell.

A

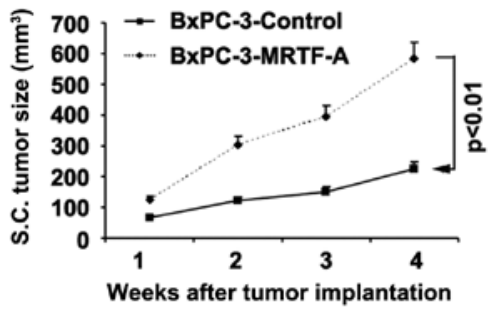

C

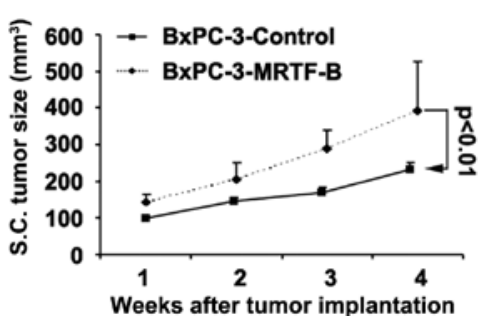

B

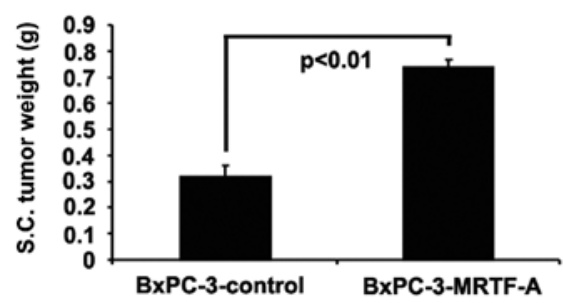

D

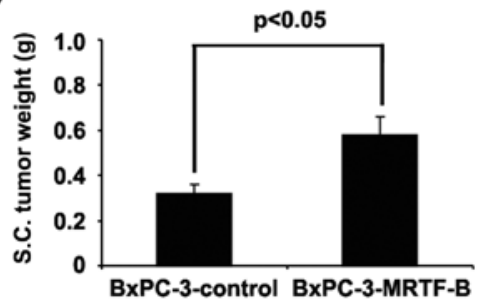

Figure 7. Effects of MRTF-A and MRTF-B on pancreatic cancer growth in the nude mouse model of s.c. xenografts. (A) BxPC-3-control and BxPC-3-MRTF-A cells $\left(1 \times 10^{6}\right)$ were inoculated into the right flank of nude mice ( $\mathrm{n}=10 /$ treatment group). Tumor size was measured weekly for 4 weeks; $n=15$. (B) Tumor weight. The above mentioned s.c. tumor was dissected, and the tumor weight was recorded; $\mathrm{n}=15$. (C) BxPC-3-control and BxPC-3-MRTF-B cells $\left(1 \times 10^{6}\right)$ were s.c. inoculated into the right flank of nude mice ( $n=10 /$ treatment group). Tumor size was measured weekly for 4 weeks as described above; $n=15$. (D) S.C. tumor weight. The above mentioned s.c. tumor (C) was dissected, and the tumor weight was recorded; $n=15$. MRTF, myocardin-related transcription factor. 
Table II. miRNA expression was significantly regulated by MRTF-A in human pancreatic cancer BxPC-3 cells.

\begin{tabular}{lcc}
\hline & $\begin{array}{c}\text { Fold change } \\
\text { miRNA }\end{array}$ & \\
\hline hsa-miR-130b-5p & -105.34 & P-value \\
hsa-miR-34 & -85.07 & $2.44 \mathrm{E}-06$ \\
hsa-miR-200 & -83.04 & $4.32 \mathrm{E}-06$ \\
hsa-let-7 & -62.85 & $2.53 \mathrm{E}-08$ \\
hsa-miR-146b-5p & -22.7 & $2.34 \mathrm{E}-06$ \\
hsa-miR-96 & -12.55 & $3.47 \mathrm{E}-09$ \\
hsa-miR-520h & -10.21 & $3.75 \mathrm{E}-13$ \\
hsa-miR-30b & -8.08 & $5.84 \mathrm{E}-10$ \\
hsa-miR-146a & -2.05 & $1.25 \mathrm{E}-21$ \\
hsa-miR-297 & 2.68 & $2.30 \mathrm{E}-20$ \\
hsa-miR-1915-3p & 2.84 & $3.56 \mathrm{E}-06$ \\
hsa-miR-665 & 3.25 & $3.73 \mathrm{E}-09$ \\
hsa-miR-1268 & 3.88 & $3.56 \mathrm{E}-08$ \\
hsa-miR-3181 & 6.05 & $5.67 \mathrm{E}-13$ \\
hsa-miR-675-3p & 7.86 & $3.90 \mathrm{E}-07$ \\
hsa-miR-155 & 10.19 & $4.30 \mathrm{E}-07$ \\
hsa-miR-21 & 10.66 & $2.28 \mathrm{E}-16$ \\
hsa-miR-221 & 21.76 & $1.18 \mathrm{E}-15$ \\
hsa-miR-203 & 63.34 & $2.21 \mathrm{E}-14$ \\
hsa-miR-210 & 134.78 & $3.05 \mathrm{E}-13$ \\
hsa-miR-612 & 150.20 & $3.34 \mathrm{E}-12$ \\
hsa-miR-222 & 170.38 & $4.53 \mathrm{E}-09$ \\
has-miR-224 & 201.89 & $3.71 \mathrm{E}-11$ \\
hsa-miR-1225-3p & 203.14 & $3.31 \mathrm{E}-11$ \\
hsa-miR-27 & 406.30 & $3.30 \mathrm{E}-10$ \\
& & \\
\hline
\end{tabular}

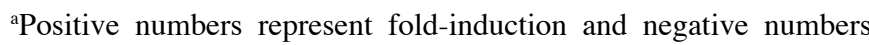
represent fold-repression; MRTF, myocardin-related transcription factor.

cells in the s.c. tumor model $(\mathrm{p}<0.01 ; \mathrm{n}=10$; Fig. $6 \mathrm{~A}$ and $\mathrm{B})$. BxPC-3-MRTF-A and BxPC-3-MRTF-B cells also significantly increased s.c. tumor weight after 4 weeks compared with BxPC-3-control cells ( $p<0.05 ; \mathrm{n}=10$; Fig. $7 \mathrm{C}$ and D). Mice given injections of BxPC-3-MRTF-A and BxPC-3-MRTF-B cells had severe skin ulcer $(90 \%)$ and gain of body weight (50\%), whereas only $30 \%$ of BxPC-3-control mice showed mild skin ulcering, but no other symptoms.

\section{Discussion}

In the present sdufy, we showed that MRTF-A and MRTF-B were upregulated in pancreatic cancer tissues and IPMN bulk tissues, compared with non-neoplastic pancreas. The results supported the hypothesis that MRTF-A and MRTF-B are oncogenes in pancreatic cancer. Leitner et al reported that MRTFs impairs migration of fibroblasts and partial knockdown of MRTFs increases motility (31). However, other studies demonstrated that MRTF double knockout in mouse embryonic fibroblasts (MEFs) impairs cell migra-
Table III. miRNA expression was significantly regulated by MRTF-B in human pancreatic cancer BxPC-3 cells.

\begin{tabular}{|c|c|c|}
\hline miRNA & $\begin{array}{c}\text { Fold change } \\
(\text { MRTF-B vs. control })^{\mathrm{a}}\end{array}$ & P-value \\
\hline hsa-miR-4304 & -99.10 & $2.54 \mathrm{E}-05$ \\
\hline hsa-miR-532-5p & -88.17 & $1.01 \mathrm{E}-03$ \\
\hline hsa-miR-1226 & -80.14 & $5.63 \mathrm{E}-03$ \\
\hline hsa-miR-34 & -69.25 & 3.67E-03 \\
\hline hsa-miR-98 & -34.79 & $1.57 \mathrm{E}-05$ \\
\hline hsa-miR-504 & -23.58 & $3.85 \mathrm{E}-13$ \\
\hline hsa-miR-451 & -18.29 & $4.63 \mathrm{E}-14$ \\
\hline hsa-miR-200 & -18.08 & $7.45 \mathrm{E}-20$ \\
\hline hsa-miR-let-7 & -3.05 & 8.40E-05 \\
\hline hsa-miR-155 & 7.68 & $2.66 \mathrm{E}-04$ \\
\hline hsa-miR-500 & 8.84 & 2.53E-06 \\
\hline hsa-miR-651 & 9.25 & $1.76 \mathrm{E}-03$ \\
\hline hsa-miR-1212 & 10.88 & $2.68 \mathrm{E}-15$ \\
\hline hsa-miR-21 & 12.15 & $1.70 \mathrm{E}-05$ \\
\hline hsa-miR-224 & 14.76 & 5.10E-04 \\
\hline hsa-miR-92b-5p & 10.89 & $2.34 \mathrm{E}-15$ \\
\hline hsa-miR-4281 & 10.96 & $2.18 \mathrm{E}-13$ \\
\hline hsa-miR-149-3p & 19.79 & $3.81 \mathrm{E}-12$ \\
\hline hsa-miR-3124 & 19.99 & $2.06 \mathrm{E}-10$ \\
\hline hsa-miR-1 & 20.98 & $1.06 \mathrm{E}-04$ \\
\hline hsa-miR-30 & 24.87 & $3.06 \mathrm{E}-06$ \\
\hline hsa-miR-10b & 26.22 & $4.06 \mathrm{E}-05$ \\
\hline hsa-miR-9 & 28.38 & $2.06 \mathrm{E}-09$ \\
\hline hsa-miR-498 & 29.98 & 4.14E-09 \\
\hline hsa-miR-612-3p & 30.21 & $5.53 \mathrm{E}-08$ \\
\hline hsa-miR-599 & 39.38 & 6.09E-06 \\
\hline hsa-miR-191 & 50.89 & 7.31E-06 \\
\hline hsa-miR-486 & 88.19 & 4.00E-08 \\
\hline hsa-miR-132 & 106.38 & $1.48 \mathrm{E}-09$ \\
\hline
\end{tabular}

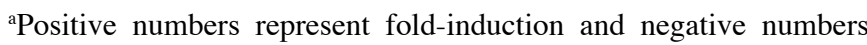
represent fold-repression; $\mathrm{N}=3$; MRTF, myocardin-related transcription factor.

tion, consistent with an important role for SRF and MRTFs in cytoskeletal organization and actin homeostasis (32) and MRTFs have been implicated in experimental metastasis, where knockdown of MRTFs reduced adhesion, migration and invasion of highly invasive cancer cells (18). Our results demonstrated that overexpression of MRTF-A and MRTF-B can promote EMT and generate stem cell-like cells in normal pancreatic cells and pancreatic cancer cells. EMT is a crucial developmental program in which immotile epithelial cells acquire mesenchymal traits. The term EMT refers to a complex molecular and cellular programme by which epithelial cells shed their differentiated characteristics, including cell-cell adhesion, planar and apical-basal polarity, and lack of motility, and acquire instead mesenchymal features, including motility, invasiveness, metastatic dissemination and an acquisition of therapeutic resistance (33-41). Importantly, 
expression of the EMT programs has been associated with poor clinical outcome in multiple tumor types (42). Although we showed that MRTF-A and MRTF-B were upregulated in pancreatic cancer tissues and they can promote EMT and generate stem cell-like cells in normal pancreatic cells and pancreatic cancer cells, we did not evaluate whether their expression was associated with poor clinical outcome. In addition, we found that MRTF-A and MRTF-B were upregulated in gemcitabine-resistant pancreatic cancer cells, implying that MRTF-A and MRTF-B could be a key molecule in gemcitabine resistance in human pancreatic cancer. They may have the potential as predictor and modulator of gemcitabine treatment.

MicroRNAs (miRs), the small non-coding RNA molecules that can suppress specific gene expression by interacting with the 3'-untranslated regions (3'-UTRs) of target mRNAs, have also been connected to EMT and cancer (43-46). EMT has been linked to stem cell phenotype $(41,47)$. We found that MRTF-A and MRTF-B could regulate many microRNAs that play an important role in pancreatic cancer. MicroRNA miR-34 inhibits human pancreatic cancer tumor-initiating cells (48); upregulation of miR-200 and let-7 leads to the reversal of EMT in gemcitabine-resistant pancreatic cancer cells (24); miRNA-96 suppresses KRAS and functions as a tumor-suppressor gene in pancreatic cancer (49); hsa-miR$520 \mathrm{~h}$ downregulates ABCG2 in pancreatic cancer cells to inhibit migration, invasion, and side populations (50); miR146a suppresses invasion of pancreatic cancer cells (51). Elevated expression of miR-155, miR-203, miR-210 and miR-222 in pancreatic tumors is associated with poorer survival (52); miR-21 is overexpressed in pancreatic cancer (53) and a potential predictor of survival; inhibition of miR-21 or miR-221 arrests the cell cycle, induces apoptosis, and sensitizes the effects of gemcitabine in pancreatic adenocarcinoma (54). We found that MRTF-A overexpression significantly downregulated miR-34, miR-200, let-7, miR-96, miR-520h and miR-146a level and evidently upregulated miR-155, miR-203, miR-210, miR-222, miR-21, miR-221 and miR-224 in pancreatic cancer cells. Similar to MRTF-A, MRTF-B could significantly inhibit miR-34 expression and upregulate miR-155, miR-21, miR-224 and miR-486 expression. Thus, we reasoned that MRTF-A and MRTF-B function as oncogenes through regulating microRNAs that play important roles in pancreatic cancer.

\section{References}

1. Jemal A, Siegel R, Xu J and Ward E: Cancer statistics, 2010. CA Cancer J Clin 60: 277-300, 2010.

2. Jemal A, Siegel R, Ward E, Hao Y, Xu J, Murray T and Thun MJ: Cancer statistics, 2008. CA Cancer J Clin 58: 71-96, 2008.

3. Li D, Xie K, Wolff R and Abbruzzese JL: Pancreatic cancer. Lancet 363: 1049-1057, 2004.

4. Olempska M, Eisenach PA, Ammerpohl O, Ungefroren $\mathrm{H}$, Fandrich $\mathrm{F}$ and Kalthoff $\mathrm{H}$ : Detection of tumor stem cell markers in pancreatic carcinoma cell lines. Hepatobiliary Pancreat Dis Int 6: 92-97, 2007.

5. Li C, Heidt DG, Dalerba P, Burant CF, Zhang L, Adsay V, Wicha M, Clarke MF and Simeone DM: Identification of pancreatic cancer stem cells. Cancer Res 67: 1030-1037, 2007.

6. Hermann PC, Huber SL, Herrler T, Aicher A, Ellwart JW, Guba M, Bruns CJ and Heeschen C: Distinct populations of cancer stem cells determine tumor growth and metastatic activity in human pancreatic cancer. Cell Stem Cell 1: 313-323, 2007.
7. Zhou BB, Zhang H, Damelin M, Geles KG, Grindley JC and Dirks PB: Tumour-initiating cells: Challenges and opportunities for anticancer drug discovery. Nat Rev Drug Discov 8: 806-823, 2009.

8. Posern G and Treisman R: Actin' together: Serum response factor, its cofactors and the link to signal transduction. Trends Cell Biol 16: 588-596, 2006.

9. Olson EN and Nordheim A: Linking actin dynamics and gene transcription to drive cellular motile functions. Nat Rev Mol Cell Biol 11: 353-365, 2010.

10. Wang DZ, Li S, Hockemeyer D, Sutherland L, Wang Z, Schratt G, Richardson JA, Nordheim A and Olson EN: Potentiation of serum response factor activity by a family of myocardin-related transcription factors. Proc Natl Acad Sci USA 99: 14855-14860, 2002.

11. Miralles F, Posern G, Zaromytidou AI and Treisman R: Actin dynamics control SRF activity by regulation of its coactivator MAL. Cell 113: 329-342, 2003.

12. Pipes GC, Creemers EE and Olson EN: The myocardin family of transcriptional coactivators: Versatile regulators of cell growth, migration, and myogenesis. Genes Dev 20: 1545-1556, 2006.

13. Goss AM, Tian Y, Cheng L, Yang J, Zhou D, Cohen ED and Morrisey EE: Wnt2 signaling is necessary and sufficient to activate the airway smooth muscle program in the lung by regulating myocardin/Mrtf-B and Fgf10 expression. Dev Biol 356: 541-552, 2011.

14. Li S, Chang S, Qi X, Richardson JA and Olson EN: Requirement of a myocardin-related transcription factor for development of mammary myoepithelial cells. Mol Cell Biol 26: 5797-5808, 2006.

15. Sun Y, Boyd K, Xu W, Ma J, Jackson CW, Fu A, Shillingford JM, Robinson GW, Hennighausen L, Hitzler JK, et al: Acute myeloid leukemia-associated Mkl1 (Mrtf-a) is a key regulator of mammary gland function. Mol Cell Biol 26: 5809-5826, 2006.

16. Morita T, Mayanagi T and Sobue K: Dual roles of myocardin-related transcription factors in epithelial mesenchymal transition via slug induction and actin remodeling. J Cell Biol 179: 1027-1042, 2007.

17. Xu WG, Shang YL, Cong XR,BianX and YuanZ: MicroRNA-135b promotes proliferation, invasion and migration of osteosarcoma cells by degrading myocardin. Int J Oncol 45: 2024-2032, 2014.

18. Medjkane S, Perez-Sanchez C, Gaggioli C, Sahai E and Treisman R: Myocardin-related transcription factors and SRF are required for cytoskeletal dynamics and experimental metastasis. Nat Cell Biol 11: 257-268, 2009.

19. Edwards BK, Brown ML, Wingo PA, Howe HL, Ward E, Ries LA, Schrag D, Jamison PM, Jemal A, Wu XC, et al: Annual report to the nation on the status of cancer, 1975-2002, featuring population-based trends in cancer treatment. J Natl Cancer Inst 97: 1407-1427, 2005.

20. Parkin DM, Bray F, Ferlay J and Pisani P: Global cancer statistics, 2002. CA Cancer J Clin 55: 74-108, 2005.

21. Perreard L, Fan C, Quackenbush JF, Mullins M, Gauthier NP, Nelson E, Mone M, Hansen H, Buys SS, Rasmussen K, et al: Classification and risk stratification of invasive breast carcinomas using a real-time quantitative RT-PCR assay. Breast Cancer Res 8: R23, 2006.

22. Yuan ZQ, Sun M,Feldman RI, Wang G, Ma X, Jiang C, Coppola D, Nicosia SV and Cheng JQ: Frequent activation of AKT2 and induction of apoptosis by inhibition of phosphoinositide-3-OH kinase/Akt pathway in human ovarian cancer. Oncogene 19: 2324-2330, 2000.

23. Maemondo M, Narumi K, Saijo Y, Usui K, Tahara M, Tazawa R, Hagiwara K, Matsumoto K, Nakamura $T$ and Nukiwa $T$ : Targeting angiogenesis and HGF function using an adenoviral vector expressing the HGF antagonist NK4 for cancer therapy. Mol Ther 5: 177-185, 2002.

24. Li Y, VandenBoom TG II, Kong D, Wang Z, Ali S, Philip PA and Sarkar FH: Up-regulation of miR-200 and let-7 by natural agents leads to the reversal of epithelial-to-mesenchymal transition in gemcitabine-resistant pancreatic cancer cells. Cancer Res 69: 6704-6712, 2009.

25. Arumugam T, Ramachandran V, Fournier KF, Wang $H$, Marquis L, Abbruzzese JL, Gallick GE, Logsdon CD, McConkey DJ and Choi W: Epithelial to mesenchymal transition contributes to drug resistance in pancreatic cancer. Cancer Res 69: 5820-5828, 2009.

26. Shimono Y, Zabala M, Cho RW, Lobo N, Dalerba P, Qian D, Diehn M, Liu H, Panula SP, Chiao E, et al: Downregulation of miRNA-200c links breast cancer stem cells with normal stem cells. Cell 138: 592-603, 2009. 
27. Wang H, Rana S, Giese N, Büchler MW and Zöller M: Tspan8, CD44 6 and alpha6beta4 are biomarkers of migrating pancreatic cancer-initiating cells. Int J Cancer 133: 416-426, 2013.

28. Li C, Wu JJ, Hynes M, Dosch J, Sarkar B, Welling TH, Pasca di Magliano M and Simeone DM: c-Met is a marker of pancreatic cancer stem cells and therapeutic target. Gastroenterology 141: 2218-2227.e5, 2011.

29. Gesierich S, Paret C, Hildebrand D, Weitz J,Zgraggen K, SchmitzWinnenthal FH, Horejsi V, Yoshie O, Herlyn D, Ashman LK, et al: Colocalization of the tetraspanins, CO-029 and CD151, with integrins in human pancreatic adenocarcinoma: Impact on cell motility. Clin Cancer Res 11: 2840-2852, 2005.

30. Yue S, Mu W and Zöller M: Tspan 8 and CD151 promote metastasis by distinct mechanisms. Eur J Cancer 49: 2934-2948, 2013.

31. Leitner L, Shaposhnikov D, Mengel A, Descot A, Julien S, Hoffmann R and Posern G: MAL/MRTF-A controls migration of non-invasive cells by upregulation of cytoskeleton-associated proteins. J Cell Sci 124: 4318-4331, 2011.

32. Mokalled MH, Johnson A, Kim Y, Oh J and Olson EN: Myocardin-related transcription factors regulate the $\mathrm{Cdk} 5 /$ Pctaire 1 kinase cascade to control neurite outgrowth, neuronal migration and brain development. Development 137: 2365-2374, 2010.

33. Tsuji T, Ibaragi S and Hu GF: Epithelial-mesenchymal transition and cell cooperativity in metastasis. Cancer Res 69: 7135-7139, 2009.

34. Larue L and Bellacosa A: Epithelial-mesenchymal transition in development and cancer: Role of phosphatidylinositol 3' kinase/AKT pathways. Oncogene 24: 7443-7454, 2005.

35. Vasioukhin V, Bauer C, Degenstein L, Wise B and Fuchs E: Hyperproliferation and defects in epithelial polarity upon conditional ablation of alpha-catenin in skin. Cell 104: 605-617, 2001.

36. Schlegelmilch K, Mohseni M, Kirak O, Pruszak J, Rodriguez JR, Zhou D, Kreger BT, Vasioukhin V, Avruch J, Brummelkamp TR, et al: Yap1 acts downstream of $\alpha$-catenin to control epidermal proliferation. Cell 144: 782-795, 2011.

37. Masszi A, Di Ciano C, Sirokmány G, Arthur WT, Rotstein OD, Wang J, McCulloch CA, Rosivall L, Mucsi I and Kapus A Central role for Rho in TGF-betal-induced alpha-smooth muscle actin expression during epithelial-mesenchymal transition. Am J Physiol Renal Physiol 284: F911-F924, 2003.

38. Cano A, Pérez-Moreno MA, Rodrigo I, Locascio A, Blanco MJ, del Barrio MG, Portillo F and Nieto MA: The transcription factor snail controls epithelial-mesenchymal transitions by repressing E-cadherin expression. Nat Cell Biol 2: 76-83, 2000.

39. Kang Y and Massagué J: Epithelial-mesenchymal transitions: Twist in development and metastasis. Cell 118: 277-279, 2004.

40. Gavert $\mathrm{N}$ and Ben-Ze'ev A: Epithelial-mesenchymal transition and the invasive potential of tumors. Trends Mol Med 14: 199-209, 2008.

41. Polyak K and Weinberg RA: Transitions between epithelial and mesenchymal states: Acquisition of malignant and stem cell traits. Nat Rev Cancer 9: 265-273, 2009.
42. Sabbah M, Emami S, Redeuilh G, Julien S, Prévost G, Zimber A, Ouelaa R, Bracke M, De Wever O and Gespach C: Molecular signature and therapeutic perspective of the epithelial-to-mesenchymal transitions in epithelial cancers. Drug Resist Updat 11: 123-151, 2008

43. Chang CJ, Chao CH, Xia W, Yang JY, Xiong Y, Li CW, Yu WH, Rehman SK, Hsu JL, Lee HH, et al: p53 regulates epithelial-mesenchymal transition and stem cell properties through modulating miRNAs. Nat Cell Biol 13: 317-323, 2011.

44. Bracken CP, Gregory PA, Kolesnikoff N, Bert AG, Wang J, Shannon MF and Goodall GJ: A double-negative feedback loop between ZEB1-SIP1 and the microRNA-200 family regulates epithelial-mesenchymal transition. Cancer Res 68: 7846-7854, 2008.

45. Gregory PA, Bracken CP, Bert AG and Goodall GJ: MicroRNAs as regulators of epithelial-mesenchymal transition. Cell Cycle 7: 3112-3118, 2008.

46. Korpal M,Lee ES, Hu G and Kang Y: The miR-200 family inhibits epithelial-mesenchymal transition and cancer cell migration by direct targeting of E-cadherin transcriptional repressors ZEB1 and ZEB2. J Biol Chem 283: 14910-14914, 2008

47. Mani SA, Guo W, Liao MJ, Eaton EN, Ayyanan A, Zhou AY, Brooks M, Reinhard F, Zhang CC, Shipitsin M, et al: The epithelial-mesenchymal transition generates cells with properties of stem cells. Cell 133: 704-715, 2008 .

48. Ji Q, Hao X, Zhang M, Tang W, Yang M, Li L, Xiang D, Desano JT, Bommer GT, Fan D, et al: MicroRNA miR-34 inhibits human pancreatic cancer tumor-initiating cells. PLoS One 4: e6816, 2009.

49. Yu S, Lu Z, Liu C, Meng Y, Ma Y, Zhao W, Liu J, Yu J and Chen J: miRNA-96 suppresses KRAS and functions as a tumor suppressor gene in pancreatic cancer. Cancer Res 70: 6015-6025, 2010.

50. Wang F, Xue X, Wei J, An Y, Yao J, Cai H, Wu J, Dai C, Qian Z, $\mathrm{Xu} \mathrm{Z}$, et al: hsa-miR-520h downregulates ABCG2 in pancreatic cancer cells to inhibit migration, invasion, and side populations. Br J Cancer 103: 567-574, 2010.

51. Li Y, Vandenboom TG II, Wang Z, Kong D, Ali S, Philip PA and Sarkar FH: miR-146a suppresses invasion of pancreatic cancer cells. Cancer Res 70: 1486-1495, 2010.

52. Greither T, Grochola LF, Udelnow A, Lautenschläger C, Würl P and Taubert H: Elevated expression of microRNAs 155, 203, 210 and 222 in pancreatic tumors is associated with poorer survival. Int J Cancer 126: 73-80, 2010.

53. Dillhoff M, Liu J, Frankel W, Croce C and Bloomston M MicroRNA-21 is overexpressed in pancreatic cancer and a potential predictor of survival. J Gastrointest Surg 12: 2171-2176, 2008.

54. Park JK, Lee EJ, Esau C and Schmittgen TD: Antisense inhibition of microRNA-21 or -221 arrests cell cycle, induces apoptosis, and sensitizes the effects of gemcitabine in pancreatic adenocarcinoma. Pancreas 38: e190-e199, 2009. 